\title{
ALIMENTOS CONSUMIDOS POR PREESCOLARES COMO COLACIÓN Y SU RELACIÓN CON EL ESTADO NUTRICIONAL
}

SNACKS EATEN BY PRE-SCHOOL CHILDREN AND ITS RELATION TO NUTRITIONAL STATE

ALIMENTOS CONSUMIDOS POR PRÉ- ESCOLARIZADOS COMO LANCHE E SUA RELAÇÃO COM O ESTADO NUTRICIONAL

\section{Resumen}

Los preescolares consumen colaciones que desempeñan un papel importante en su alimentación e influyendo en los hábitos alimentarios. Objetivo: Conocer la selección de alimentos consumidos por preescolares durante la colación, pautada o no por la Institución y su relación con el Estado Nutricional. Métodos: Se evaluó Estado Nutricional según IMC/Edad, se registró los alimentos consumidos y su aceptación. Se utilizó Chi2 Resultados: El $62 \%$ de los niños presentó IMC/Edad normal, 21\% sobrepeso y $16 \%$ obesidad. Los grupos de alimentos más consumidos fueron azúcares/dulces, cereales/legumbres

Oberto María G. ${ }^{1}$ Schiaroli Jessica A. ${ }^{1}$ Lencina Rocío del Cielo. ${ }^{1}$

Sosa Mariela I. ${ }^{1}$ Sanchéz Roberto J. ${ }^{1}$

${ }^{1}$ Escuela de Nutrición. FCM/UNC. georginaoberto@hotmail. com

Trabajo recibido: 22 de Noviembre 2017.

Aprobado: 21 de Marzo 2017. y aceites/grasas. Existe asociación estadísticamente significativa entre la aceptación de alimentos saludables en preescolares con la Institución a la que asisten $(\mathrm{p}<0,05)$. No existe asociación estadísticamente significativa entre los alimentos saludables consumidos por preescolares y su IMC/Edad ( $p>0,05)$. Conclusiones: Las Instituciones que pautan colaciones intentan promover una alimentación más saludable, sin embargo los preescolares consumen gran cantidad de alimentos poco saludables, independientemente de su estado nutricional.

Palabras clave: preescolares, alimentos saludables, estado nutricional 


\section{Abstract}

Pre-school children eat snacks which have an important role in their feeding and influence their eating habits. Objective: To know about the selection of food eaten by pre-school children as snacks, regulated by the institution or not, and its relation to their Nutritional State. Methods: Nutritional State was assessed according to BMI/Age, the food eaten and its acceptance were registered. Chi2 Results were used: $62 \%$ of the children presented normal BMI/Age, $21 \%$ overweight, and $16 \%$ obesity. The most eaten food groups were sugar/sweets, cereals/legumes and oil/fats. Statistically, there is a significant association between acceptance of healthy food in pre-school children and the institution they attend $(p<0,05)$. There is no statistically significant association between healthy food eaten by pre-school children and their BMI/Age $(p>0,05)$. Conclusions: Institutions which regulate snacks try to promote healthier eating habits; however pre-school children eat a great quantity of unhealthy food, independent of their nutritional state.

Key words: pre-school children, healthy food, nutritional state

\section{Resumo}

Pré-escolarizados consomem lanches que desempenham um papel importante na sua alimentação e que influem nos hábitos alimentares. Objetivo: Conhecer a seleção de alimentos consumidos pelos pré-escolares como lanche, programada ou não pela Instituição e sua relação com o Estado Nutricional. Métodos: O Estado Nutricional foi avaliado segundo o IMC / Idade, os alimentos consumidos e sua aceitação foram registrados. Resultados Utilizou-se o Chi2: $62 \%$ de crianças evidenciou IMC / Idade normal, $21 \%$ sobrepeso e $16 \%$ obesidade. Os segmentos de alimentos mais consumidos foram açúcar / doces, cereais / legumes e óleos / gorduras. Existe uma associação estatisticamente significativa entre a aceitação de alimentos salutares em pré-escolarizados com a Instituição receptora $(\mathrm{p}<0,05)$. Não há associação estatisticamente significativa entre alimentos salutares consumidos por pré-escolares e seu IMC / Idade ( $>>0,05)$. Conclusões: As Instituições que orientam sobre os lanches tentam promover uma dieta mais salutar, contudo, os pré-escolares consomem grandes quantidades de alimentos pouco salutares, independentemente de seus estados nutricionais.

Palavras-chave: pré-escolar, saúde alimentos, estado nutricional

\section{Introducción}

El niño en edad preescolar comienza a introducirse en una amplia gama de oferta alimentaria, pasa de depender casi exclusivamente de sus padres y cuidadores a comer progresivamente solo. En esta etapa se da con mayor profundidad la construcción de los hábitos, patrones aprendidos de naturaleza compleja, que al inicio son voluntarios y luego se tornan involuntarios, también ocurren cambios en el consumo de alimentos ya sea desde los gustos hasta los tipos y la cantidad (1).

Si se analizan los alimentos que consumen los preescolares, en cuanto a la cantidad, no presentan deficiencias en la mayoría de los casos. Sin embargo, se puede observar que la calidad de la dieta suele ser el problema que enfrentan estos niños, mostrando que la frecuencia de consumo de frutas y verduras es baja, con un elevado consumo de alimentos como azúcar y dulces, golosinas y snacks (2).

Actualmente, la mayoría de los niños comienza a asistir a Instituciones de Enseñanza Preescolar (Jardines maternales y Jardines de infantes) en los cuales pasan varias horas al día. En ellos realizan comidas proporcionalmente más pequeñas que las comidas principales (colación), forman parte de la alimentación diaria e influyen tanto en el Estado 
Nutricional como en la adquisición de hábitos alimentarios del niño (3).

En algunas Instituciones de Enseñanza Preescolar Privadas, los directivos establecen una colación pautada según criterios propios, la cual consiste en solicitar a los padres los alimentos que deben llevar sus hijos como colación cada día de la semana, determinando así la elección de los mismos; si bien los criterios adoptados por los directivos no provienen de las recomendaciones de Profesionales de la Nutrición, intentan promover el consumo de una alimentación más variada y nutritiva.

Muchas veces las colaciones no suelen ser las más adecuadas y están constituidas por alimentos industrializados con alta densidad energética, gran contenido de azúcar, grasas saturadas, sodio y carentes en vitaminas, minerales y fibra $(4,5)$. Por esa razón, el estudio estuvo orientado a conocer los alimentos que consumen los niños durante la colación, pautada o no pautada por la Institución, y su relación con el Estado Nutricional en dos Establecimientos Privados de Enseñanza Preescolar de la ciudad de Córdoba en el año 2015.

\section{Materiales y Métodos}

Se realizó un estudio observacional con carácter descriptivo simple y comparativo, transversal. La muestra estuvo constituida por niños preescolares de ambos sexos que asistieron con regularidad, los 5 días de la semana en que se realizó la recolección de datos, a las Instituciones Privadas de Enseñanza Preescolar Chispitas, perteneciente al Instituto Francisco Luis Bernardez y Divino Niño Jesús, perteneciente al Instituto Juan XXIII con colación pautada y sin colación pautada respectivamente (6-8).

Durante el desarrollo de la Investigación se respetaron las recomendaciones de la Declaración de Helsinki y las Normas CIOMS $(8,9)$. Para la implementación se contó con el aval de los directivos de las Instituciones, quienes comunicaron a los padres/tutores de los niños participantes, los cuales firmaron el consentimiento informado. Se excluyeron los preescolares cuyos cuidadores no firmaron el consentimiento, además en el trabajo de campo se consideró el asentimiento de los niños.

Las variables en estudio quedaron comprendidas por sexo, edad, estado Nutricional, colación pautada o no pautada por la Institución, alimentos que se consumen durante la colación y aceptación de alimentos en los niños.

Para la variable alimentos que se consumen durante la colación se utilizaron dos indicadores: alimentos saludables consumidos como colación, entendidos como "Toda sustancia o mezcla de sustancias que aporta nutrientes esenciales y energía para mantenerse sano" y alimentos poco saludables consumidos como colación, definidos como " Toda sustancia o mezcla de sustancias densas en energía con alto contenido de azúcar, sal, grasas y con bajo aporte de nutrientes en términos de proteínas, fibras, vitaminas y minerales" $(4,5)$. Los mismos se agruparon de la siguiente forma:

Más alimentos saludables: niños que consumen más de 5 alimentos saludables y menos de 3 poco saludables como colación durante una semana. Iguales en cantidad: niños que consumen 4 alimentos saludables y 4 poco saludables como colación durante una semana. Más alimentos poco saludables: niños que consumen menos de 3 alimentos saludables y más de 5 poco saludables como colación durante una semana $(4,5,10)$.

Se diseñó una guía de observación, donde se registraron los alimentos y bebidas que consumieron los niños a lo largo de la semana durante su colación pautada o no pautada por la Institución. El procedimiento se llevó a cabo al horario de la colación durante 5 días seguidos de la semana, el equipo, dividido por sala, registró los alimentos y bebidas consumidos por los niños, identificándolos por su nombre en el pintor, mantel o mochila $(11,12)$.

Los niños se pesaron con un mínimo de ropa y sin calzado en una balanza de pie digital marca "Gama Italy Profesional" con sensibilidad de 100g. Se colocó al niño en el centro de la plataforma y se efectuó la lectura. 
Para la talla, se midieron los niños de pie y de espalda a la pared, sin zapatos, con la protuberancia occipital, las nalgas y sus talones tocando la pared, los pies bien asentados en el suelo con los talones juntos formando un ángulo de 45 grados; con una cinta métrica pegada a la pared sin zócalo, conjuntamente se utilizó una escuadra de madera para deslizarla en la pared hasta la cabeza. Se le pidió al niño que haga una inspiración profunda y mantenga la posición erguida para realizar la lectura en centímetros, en el plomo de la cinta métrica que coincidió en el vértice del ángulo recto de la escuadra. Se contó con dos personas para realizar la medición, una de ellas colocó una mano sobre los pies del niño para evitar que levantara los talones, y la otra mano sobre las rodillas para asegurar que estuvieran bien estiradas, la otra persona aplicó tracción ligera a la cabeza del niño, hacia arriba, a partir de la apófisis mastoidea y colocó la escuadra para efectuar la lectura (13-15).

Los datos antropométricos fueron analizados con el indicador índice de masa corporal (IMC/Edad) según las "Curvas de crecimiento de la OMS 2006”, permitiendo determinar el Estado Nutricional de los niños en Eutróficos o Malnutrición por exceso (sobrepeso y obesidad) (16).

Los valores obtenidos de la guía de observación y el análisis medicional fueron cargados en una base de datos en Microsoft Office Excel 2010 y SPSS. La técnica estadística utilizada fue prueba Chi Cuadrado con un nivel de significación de 0,05 y un nivel de confianza de un $95 \%$.

\section{Resultados:}

La muestra estuvo conformada por 212 niños de 2 a 5 años, con una media de 4,9 años, el $46 \%(n=98)$ de sexo masculino y el restante $54 \%(n=114)$ de sexo femenino.

El 37\% de los niños pertenecen a la Institución de Enseñanza Preescolar sin colación pautada (ISCP) y el 63\% a la Institución de Enseñanza Preescolar con colación pautada (ICP)

En la tabla 1 se observa que la mayoría de los niños eran eutróficos (62\%). Un 21\% presentó sobrepeso y un $16 \%$ obesidad.

Tabla Nº1: Distribución porcentual del Estado Nutricional según IMC/Edad en ambas Instituciones Privadas de Enseñanza Preescolar

\begin{tabular}{|l|l|l|}
\hline $\begin{array}{l}\text { ESTADO NUTRICIONAL } \\
\text { SEGÚN IMC }\end{array}$ & $\mathrm{n}$ & $\%$ \\
\hline OBESIDAD & 41 & $16 \%$ \\
\hline SOBREPESO & 56 & $21 \%$ \\
\hline EUTRÓFICO & 177 & $62 \%$ \\
\hline RIESGO DE BAJO PESO & 2 & $1 \%$ \\
\hline BAJO PESO & 0 & $0 \%$ \\
\hline TOTAL & 276 & $100 \%$ \\
\hline
\end{tabular}

En relación al grupo de alimentos consumidos como colación en la ICP en su mayoría pertenecen al grupo de cereales y legumbres (31\%), seguido por azúcares y dulces (24\%), continuando con leche, yogur y quesos $(18 \%)$.

En la ISCP en su mayoría los alimentos consumidos pertenecen a los grupos de azúcares y dulces (35\%), seguido por aceites y grasas (30\%), continuando con cereales y legumbres $(19 \%)$. 
En relación a los alimentos consumidos como colación durante una semana, se observó que en la ICP los alimentos más consumidos fueron frutas $(11,7 \%)$, seguido de sándwich de jamón y queso, pebete y sándwich de milanesa $(10,2 \%)$, continuando con cereales azucarados o de chocolate, copos, almohadas, barra de cereal, tutucas, mix cereales, pochoclo $(9,4 \%)$.

En lo que respecta la ISCP los alimentos más consumidos fueron galletas de chocolate rellenas, tipo bizcocho de grasa $(13,2 \%)$, seguido de alfajor de chocolate (simple, triple), obleas de chocolate $(10,4 \%)$, continuando con galletas simples, de agua, salvado, integral, obleas, vainillas, chalitas $(7,9 \%)$.

En ambas Instituciones se observó que las bebidas más consumidas fueron jugos concentrados, gaseosas y agua saborizada con o $\sin$ gas $(17,5 \%)$, teniendo un bajo consumo de agua $(11,2 \%)$.

En la tabla 2 se observa que las variables tienen un alto nivel de dependencia $(\mathrm{p}=0,001)$. Los niños más pequeños ingieren mayor cantidad de colaciones saludables que poco saludable $(45 \%)$. En los más grandes, esta relación se invierte, consumiendo mayor cantidad de alimentos poco saludables (51\%).

Tabla N²: Distribución porcentual del consumo de alimentos saludables en ambas Instituciones Privadas de Enseñanza Preescolar según rango de edad.

\begin{tabular}{|c|c|c|c|c|c|c|c|c|}
\hline \multirow{3}{*}{ EDAD } & \multicolumn{8}{|c|}{ CONSUMO DE ALIMENTOS } \\
\hline & \multicolumn{2}{|c|}{$\begin{array}{c}\text { Más } \\
\text { alimentos } \\
\text { saludables }\end{array}$} & \multicolumn{2}{|c|}{$\begin{array}{l}\text { Igual en } \\
\text { cantidad }\end{array}$} & \multicolumn{2}{|c|}{$\begin{array}{c}\text { Más alimentos } \\
\text { poco } \\
\text { saludables }\end{array}$} & \multicolumn{2}{|c|}{ Total } \\
\hline & $n$ & $\%$ & $\bar{n}$ & $\%$ & $\bar{n}$ & $\%$ & $\bar{n}$ & $\%$ \\
\hline $\begin{array}{c}<4 \text { años y } \\
\text { medio }\end{array}$ & 50 & $46 \%$ & 6 & $23 \%$ & 21 & $27 \%$ & 77 & $36 \%$ \\
\hline $\begin{array}{c}4 \text { años y } \\
\text { medio a } 5 \\
\text { años y } \\
\text { medio }\end{array}$ & 34 & $30 \%$ & 9 & $35 \%$ & 17 & $22 \%$ & 60 & $28 \%$ \\
\hline $\begin{array}{c}>5 \text { años y } \\
\text { medio }\end{array}$ & 24 & $22 \%$ & 11 & $42 \%$ & 40 & $51 \%$ & 75 & $35 \%$ \\
\hline Total & 108 & $100 \%$ & 26 & $100 \%$ & 78 & $100 \%$ & 212 & $100 \%$ \\
\hline
\end{tabular}

En la tabla 3 se constata que el nivel de aceptación no se distribuye del mismo modo entre niños y niñas $(p=0,036)$. En cuanto a la aceptación alta de alimentos saludables las niñas presentan un porcentaje mayor (62\%) que los niños (38\%).

Con respecto a la aceptación regular de alimentos saludables los niños presentan un mayor porcentaje (55\%) que las niñas (45\%). En cuanto a la aceptación baja de alimentos saludables presentan pocas variaciones entre sexos, con un $57 \%$ los niños y un $43 \%$ las niñas. 
Tabla No3: Distribución porcentual en la aceptación de alimentos saludables en ambas Instituciones de Enseñanza Preescolar según sexo.

\begin{tabular}{|l|l|c|c|c|c|c|c|c|}
\hline \multirow{3}{*}{\begin{tabular}{c} 
SEXO \\
\cline { 2 - 10 }
\end{tabular}} & \multicolumn{2}{|c|}{$\begin{array}{c}\text { ALTA } \\
\text { ACEPTACIÓN }\end{array}$} & $\begin{array}{c}\text { REGULAR } \\
\text { ACEPTACIÓN }\end{array}$ & \multicolumn{2}{c|}{$\begin{array}{c}\text { BAJA } \\
\text { ACEPTACIÓN }\end{array}$} & \multicolumn{2}{c|}{} \\
\cline { 2 - 10 } & $\mathrm{n}$ & $\%$ & $\mathrm{n}$ & $\%$ & $\mathrm{n}$ & $\%$ & $\mathrm{~N}$ & $\%$ \\
\hline Masculino & 57 & $39 \%$ & 54 & $56 \%$ & 18 & $51 \%$ & 129 & $47 \%$ \\
\hline Femenino & 88 & $61 \%$ & 42 & $44 \%$ & 17 & $49 \%$ & 147 & $53 \%$ \\
\hline Total & 145 & $100 \%$ & 96 & $100 \%$ & 35 & $100 \%$ & 276 & $100 \%$ \\
\hline
\end{tabular}

En la tabla 4 se visualiza que el nivel de aceptación no se distribuye del mismo modo. A medida que los preescolares crecen, se acentúa la baja aceptación de alimentos saludables $(\mathrm{p}=0,019)$.

Con respecto a la aceptación alta de alimentos saludables los niños menores de 4 años y medio presentan un mayor porcentaje (41\%). Con respecto a la aceptación regular de alimentos saludables los niños de 4 años y medio a 5 años y medio presentan un mayor porcentaje ( $42 \%$ ). En cuanto a la aceptación baja de alimentos saludables el porcentaje más elevado es el de los niños de 5 años y medio (50\%).

Tabla Nº: Distribución porcentual en la aceptación de alimentos saludables en ambas Instituciones de Enseñanza Preescolar según rango de edad.

\begin{tabular}{|l|l|l|c|c|c|c|c|c|}
\hline \multirow{3}{*}{ EDAD } & \multicolumn{7}{|c|}{ ACEPTACIÓN DE ALIMENTOS } \\
\cline { 2 - 9 } & \multicolumn{2}{|c|}{$\begin{array}{c}\text { ALTA } \\
\text { ACEPTACIÓN }\end{array}$} & $\begin{array}{c}\text { REGULAR } \\
\text { ACEPTACIÓN }\end{array}$ & $\begin{array}{c}\text { BAJA } \\
\text { ACEPTACIÓN }\end{array}$ & \multicolumn{2}{c|}{ TOTAL } \\
\cline { 2 - 9 } & $\mathrm{n}$ & $\%$ & $\mathrm{n}$ & $\%$ & $\mathrm{n}$ & $\%$ & $\mathrm{n}$ & $\%$ \\
\hline$<4$ años & 46 & $41 \%$ & 20 & $28 \%$ & 10 & $33 \%$ & 76 & $36 \%$ \\
\hline $\begin{array}{l}4 \text { años y } \\
\text { medio a } \\
5 \text { años y } \\
\text { medio }\end{array}$ & 26 & $23 \%$ & 30 & $42 \%$ & 5 & $17 \%$ & 61 & $29 \%$ \\
\hline $\begin{array}{l}>5 \text { años } \\
\text { y medio }\end{array}$ & 39 & $35 \%$ & 21 & $30 \%$ & 15 & $50 \%$ & 75 & $35 \%$ \\
\hline Total & 111 & $100 \%$ & 71 & $100 \%$ & 30 & $100 \%$ & 212 & $100 \%$ \\
\hline
\end{tabular}

Al analizar la relación entre la aceptación de alimentos saludables de los niños y la Institución de Enseñanza Preescolar a la que asisten, las preferencias en el consumo de alimentos saludables se distribuyen de distinta manera.

Entre los niños que tienen aceptación alta de alimentos saludables, el $83 \%$ pertenecen a la ICP. Por el contrario, en el grupo de niños con baja aceptación de alimentos saludables, el $100 \%$ pertenece a la ISCP.

En la tabla 5 se observa que de los niños eutróficos el $28 \%$ consume alimentos de los grupos azúcares y dulces, el $25 \%$ cereales y legumbres, el 22\% aceites y grasas y en menor medida agua, leche queso y yogur, frutas y verduras. De los niños con malnutrición por exceso (sobrepeso y obesidad), el 27\% eligió alimentos del grupo de cereales y legumbres, 
el $26 \%$ azúcares y dulces, continuando con el $20 \%$ aceites y grasas, y en menor medida de agua, leche queso y yogur, frutas y verduras.

Tabla N5: Distribución porcentual de los grupos de alimentos consumidos según IMC/ Edad en ambas Instituciones Privadas de Enseñanza Preescolar

\begin{tabular}{|l|c|c|c|c|c|c|}
\hline \multirow{2}{*}{$\begin{array}{l}\text { Grupos de } \\
\text { alimentos }\end{array}$} & \multicolumn{2}{|c|}{$\begin{array}{c}\text { MALNUTRICIÓN } \\
\text { POR EXCESO }\end{array}$} & \multicolumn{2}{|c|}{ EUTRÓFICO } & \multicolumn{2}{c|}{ Total } \\
\cline { 2 - 7 } & $\mathrm{N}$ & $\%$ & $\mathrm{n}$ & $\%$ & $\mathrm{~N}$ & $\%$ \\
\hline $\begin{array}{l}\text { Cereales y } \\
\text { legumbres }\end{array}$ & 188 & $27 \%$ & 306 & $25 \%$ & 494 & $26 \%$ \\
\hline $\begin{array}{l}\text { Frutas y } \\
\text { verduras }\end{array}$ & 52 & $8 \%$ & 84 & $7 \%$ & 136 & $7 \%$ \\
\hline $\begin{array}{l}\text { Leche queso } \\
\text { yogur }\end{array}$ & 51 & $7 \%$ & 82 & $7 \%$ & 133 & $7 \%$ \\
\hline Aceites y grasas & 134 & $20 \%$ & 263 & $22 \%$ & 397 & $21 \%$ \\
\hline $\begin{array}{l}\text { Azúcares y } \\
\text { dulces }\end{array}$ & 179 & $26 \%$ & 331 & $28 \%$ & 510 & $27 \%$ \\
\hline Agua & 80 & $12 \%$ & 137 & $11 \%$ & 217 & $11 \%$ \\
\hline TOTAL & 684 & $100 \%$ & 1203 & $100 \%$ & 1887 & $100 \%$ \\
\hline
\end{tabular}

En la tabla 6 se observa que la distribución de alimentos saludables que consumen los niños no se relaciona con el Estado Nutricional de los mismos $(p=0,492)$. Los niños eutróficos consumen igual cantidad de alimentos saludables que poco saludables.

Tabla No6: Distribución porcentual de alimentos saludables consumidos según Estado Nutricional en ambas Instituciones Privadas de Enseñanza Preescolar

\begin{tabular}{|c|c|c|c|c|c|c|c|c|}
\hline \multirow{3}{*}{$\begin{array}{c}\text { ESTADO } \\
\text { NUTRICIONAL } \\
\text { SEGÚN IMC/Edad }\end{array}$} & \multicolumn{8}{|c|}{ CONSUMO DE ALIMENTOS SEMANAL } \\
\hline & \multicolumn{2}{|c|}{$\begin{array}{l}\text { Más alimentos } \\
\text { saludables }\end{array}$} & \multicolumn{2}{|c|}{$\begin{array}{l}\text { Igual en } \\
\text { cantidad }\end{array}$} & \multicolumn{2}{|c|}{$\begin{array}{l}\text { Más alimentos } \\
\text { poco } \\
\text { saludables }\end{array}$} & \multicolumn{2}{|c|}{ Total } \\
\hline & $n$ & $\%$ & $\mathrm{n}$ & $\%$ & $\mathrm{n}$ & $\%$ & $n$ & $\%$ \\
\hline EUTRÓFICO & 67 & $60 \%$ & 19 & $83 \%$ & 46 & $61 \%$ & 132 & $63 \%$ \\
\hline $\begin{array}{l}\text { MALNUTRICIÓN POR } \\
\text { EXCESO }\end{array}$ & 44 & $40 \%$ & 4 & $17 \%$ & 30 & $39 \%$ & 78 & $37 \%$ \\
\hline Total & 111 & $100 \%$ & 23 & $100 \%$ & 76 & $100 \%$ & 210 & $100 \%$ \\
\hline
\end{tabular}

\section{Discusión}

Con respecto a la relación entre la Aceptación de alimentos y la Institución a la que asisten los niños, se observó que de la aceptación alta de alimentos saludables el 83\% de los niños pertenece a la ICP y el 17\% a la ISCP, en la aceptación regular de alimentos saludables el $59 \%$ pertenece a la ICP y el $41 \%$ a la ISCP y en la aceptación baja de alimentos saludables el $100 \%$ de los niños pertenece a la ISCP.

En un estudio realizado en Chile, se observó la influencia del kiosco saludable en la elección de alimentos por parte de los escolares, revelando que los niños luego de la implementación del kiosco saludable eligieron como colaciones frutas, leche, yogur, jugos bajos en calorías, sándwich de verdura, frutas secas y helados de agua, a diferencia de la escuela sin kiosco saludable, en la que aumentaron las preferencias por los snacks salados 
y dulces y bebidas azucaradas (17). Se encontraron resultados semejantes en otro estudio en donde se comparan las colaciones de los preescolares con colación pautada y las de los escolares sin colación pautada, encontrando que existe una mayor aceptación de alimentos saludables en preescolares ( $90 \%$ llevan colaciones saludables 3 o más veces a la semana) que escolares (64\%) (18).

En lo que respecta a la relación de Alimentos consumidos como colación y el Estado Nutricional de los niños bajo estudio, se encontró que la distribución de alimentos saludables que consumen, no se relaciona con el Estado Nutricional de los mismos. Similares resultados poseen los estudios de Coromoto Nava M y col. en donde se analizó la relación entre el estado antropométrico, hábitos alimentarios y nivel de actividad física en preescolares, obteniendo como resultado que no existe asociación entre hábitos alimentarios y Estado Nutricional antropométrico (19). Según resultados de otros autores los hábitos alimentarios de los niños no son saludables en general, independientemente de la presencia de sobrepeso, obesidad o un Estado Nutricional normal (20-22). A diferencia de un estudio realizado en México, en el que concluyeron que podría existir un vínculo entre el estado nutricional y el aporte calórico de los refrigerios, puesto que la mayoría de los preescolares se hallaron con un estado nutricional normal mientras que el contenido calórico de sus colaciones se localizó en rangos adecuados de aporte calórico (23).

\section{Conclusiones}

En nuestra investigación se pone de manifiesto un frecuente consumo de alimentos poco saludables en las colaciones, independientemente del Estado Nutricional de los preescolares. Predominan alimentos con alto contenido de azúcares, grasas, sal y carente en valor nutritivo, que afectan de forma negativa la salud de los niños y refuerzan hábitos alimentarios poco saludables.

Si bien la colación en la Institución, por ser una única comida diaria, no determinaría el Estado Nutricional de los niños, es un espacio que debe ser aprovechado para la construcción de hábitos alimentarios saludables (22).

Consideramos importante que la comunidad educativa promocione prácticas saludables de alimentación, trabajando en conjunto con profesionales de la nutrición y los demás actores implicados en la creación de entornos saludables dentro de las Instituciones, que fomenten la continuidad de estas prácticas en los niños de todas las edades, a través de kioscos Saludables, Educación Alimentaria Nutricional entre otras.

Como profesionales de la salud es nuestra competencia intervenir de manera apropiada en la planificación, elaboración y acción de estrategias y recomendaciones que contribuyan a la creación de hábitos alimentarios saludables de los niños y su entorno, para mejorar su calidad de vida y evitar riesgos en el futuro.

\section{Agradecimientos}

A los directivos y docentes de las Instituciones educativas por su disposición.

A Prof. Dra. Patricia Quiroga por su asesoramiento y revisión del manuscrito final. 
1. Grande MC, Román MD. Nutrición y Salud Materno Infantil. Córdoba, Argentina: Brujas; 2014.

2. Britos S, Pueyrredón P, O»Donnell A. Cap 5: Comer en una edad difícil de $1 \mathrm{a}$ 4 años. En: ¿Cómo comen los niños entre 1 a 4 años en la argentina? Argentina: CESNI. 2006. [citado 30 de octubre de 2015]. Disponible en: http://www.cesni.org. ar/sistema/archivos/183-comocomenlibro.pdf.

3. Plazas M, Johnson S. Nutrición del preescolar y el escolar. En: Casanueva E., Kaufer-Horwitz M, Pérez-Lizaur AB, et al. Nutriología Médica. $3^{\text {a }}$ ed. México: Médica Panamericana; 2008. p.77-118.

4. Espínola V y Brünner M. Rol de la escuela en la formación de una alimentación sana. En su: Alimentación saludable en la escuela. Hacia una adecuada alimentación de los niños en edad escolar. $1^{\mathrm{a}}$ ed. Ministerio de Educación. Ciudad Autónoma de Buenos Aires.2014. p. 23-30.

5. Bustos NZ. ¿Qué colación le envío a mi hijo? Revista Nutrición y Vida. Ed. N 8. 2014. [citado Dic 15 de diciembre de 2014]. Disponible en: http://issuu.com/ carlosdonaire/docs/papel_digital_nyv_8.

6. Pineda EB, Alvarado LE. Diseño para investigación cuantitativa. En su: Metodología de la Investigación. OPS. Washington D.C. 2008. p. 79-99.

7. Sabulsky J. Selección del tipo de estudio. En su: Investigación científica en saludenfermedad. Córdoba, Argentina: Triunfar; 2002. p. 123-165.

8. Asociación Médica Mundial. Declaración de Helsinki. Principios éticos para las investigaciones médicas en seres humanos. Helsinki, Finlandia. 1964.

9. Consejo de Organizaciones Internacionales de las Ciencias Médicas. Pautas éticas internacionales para la Investigación biomédica en seres humanos. CIOMS, Ginebra. 2002.

10. FAO (Organización de las Naciones Unidas para la agricultura y la alimentación). Guía de nutrición de la familia. Roma. 2006

11. Cameron ME, van Staveren WA. Manual on methodology for food consumption studies. Oxford: Oxford University Press, 1988.

12. Sabaté J. Estimación de la ingesta dietética: métodos y desafíos. Med Clínica (Barc), 1993; 100:591-596.

13. Carlos Aibar Remón C, Rabanaque MJ, Alvarez-Dardet C, Nolasco A, Mancho J y Gascón E. Evolución de los diseños epidemiológicos de la investigación clínica en España (1975-1994). Rev Esp Salud Pública. 1999; 73: 445-453

14. Serra M, Román B y Ribas L. Metodología de los estudios nutricionales. Actividad dietética 12 (2001): 180-184.

15. Sociedad Argentina de Pediatría. Guía para la evaluación del crecimiento físico. $3^{\mathrm{a}}$ ed. Ciudad Autónoma de Buenos Aires, Argentina: Ideográfica; 2013. 138p.

16. Evaluación del estado nutricional de niñas, niños y embarazadas mediante antropometría. Elvira Calvo et al. $1^{\mathrm{a}}$ ed. Buenos Aires, Argentina: Ministerio de Salud de la Nación, 2009. 144p.

17. Bustos N, Kain J, Leyton B, Vio F. Cambios en el patrón de consumo de alimentos en escolares chilenos con la implementación de un kiosco saludable. ALAN. 2011; 61(3): 302-307.

18. Crovetto M, Zamorano N, Medinelli A. Estado nutricional, conocimientos y conductas en escolares de kinder y primer año básico en 3 escuelas focalizadas por obesidad infantil en la comuna de Valparaíso, Chile. Rev chil nutr. 2010; 37(3): 309320 .

19. Coromoto Nava BM, Pérez A, Herrera HA, Hernández HR. Hábitos alimentarios, actividad física y su relación con el estado nutricional-antropométrico de 
preescolares. Rev. chil. nutr. 2011; 38(3): 301-312.

20. Medina Montaño R, Moreno Pérez V, Romero Velarde E. Comparación del consumo de alimentos durante el horario escolar en niños de 6-11 años de edad con sobrepeso, obesidad y peso saludable. RESPIN. 2012; 13(3).

21. Rapp, Kilian, et al. «Type of kindergarten and other potential determinants of overweight in pre-school children.» Public Health Nutrition-Wallingford 8.6 (2005): 642-649.

22. Atkin L, Davies P. Diet composition and body mass index in pre-school children. Am J Clin Nutr. 2000; 72 (1): 15-21.

23. Valdés Santillana A L. Relación entre el estado nutricio y el aporte calórico del contenido alimentario en los refrigerios de los preescolares del Jardín de niños Rosario Castellanas de la población de la $2^{\mathrm{a}}$ Manzana Santa Cruz Tepexpan, Jiquipilco [tesis].Toluca, México ; 2013. 